\title{
Multilayer Graph Edge Bundling
}

\author{
Romain Bourqui* \\ LaBRI \\ Université de Bordeaux \\ France
}

\author{
Dino lenco ${ }^{\dagger}$ \\ IRSTEA - UMR TETIS \\ Montpellier \\ France
}

\author{
Arnaud Sallaberry \\ LIRMM - Université Paul Valéry \\ Montpellier \\ France
}

\author{
Pascal Poncelet ${ }^{\S}$ \\ LIRMM \\ Université de Montpellier \\ France
}

\begin{abstract}
Many real world information can be represented by a graph with a set of nodes interconnected with each other by multiple type of relations called edge layers (e.g., social network, biological data). Edge bundling techniques have been proposed to solve cluttering issue for standard graphs while few efforts were done to deal with the similar issue for multilayer graphs. In multilayer graphs scenario, not only the clutter induced by large amount of edges is a problem but also the fact that different type of edges can overlap each other making useless the final visualization. In this paper we introduce a new multilayer graph edge bundling technique that firstly produces a preliminary edge bundling independently of the different edge layers and then deals with the specificity of multilayer graphs where more than one type of edges can be routed on the same bundle. The proposed visualization is tested on a real world case study and the outcomes point out the ability of our proposal to discover patterns present in the data.
\end{abstract}

Index Terms: I.3.3 [Computer Graphics]: Picture/Image Generation-Line and curve generation;

\section{INTRODUCTION}

Nowadays many types of data exhibit complex relational structures. For instance, by considering different social networks spanning over the same set of people, but with different life aspects (e.g. social relationships such as Facebook, Twitter, LinkedIn, etc.), we can get as many relation types as the different aspects. In biology, protein-protein interaction networks can be created considering the pairs of proteins that have direct interaction, physical association or they are co-localised [24]. More examples can be quoted from a gene network where genes are connected by considering the different pathway interactions and recommendation networks [15].

These data require a structure to support the representation of multiple relations among entities. A structure can fit these characteristic is the multilayer graph. A multilayer graph is defined as a graph, with the additional features that more than one edge can exist between the same pair of nodes and each edge may have a different type. Semantically speaking, considering the social network scenario, each edge type (or layer) can be interpreted as a particular social interaction between individuals. For example, a layer can represent interactions coming from the Facebook social network, another layer can represent interactions coming from LinkedIn and so on. Formally, given a set of layers $L=\left\{L_{1}, \ldots, L_{d}\right\}$, a multilayer graph $G$ is defined as a tuple $\left(V,\left\{E_{i}\right\}_{i=1}^{|L|}, L\right)$ where, $V$ is the set of vertices, $E_{i} \subseteq V \times V$ is the set of undirected edges over dimension $L_{i} \in L$.

Such a rich model introduces the possibility to represent more fine-grained information thanks to the multi-layer structure; on the

\footnotetext{
*e-mail: romain.bourqui@labri.fr

†e-mail: dino.ienco@teledetection.fr

†e-mail: arnaud.sallaberry@lirmm.fr

§e-mail: pascal.poncelet@lirmm.fr
}

other hand this extra information needs the definition of new visual tasks involving the analysis of the correlation among layers: In which layer(s) a community of nodes appears? What are the common patterns between layers? Which are the specific patterns of a layer w.r.t. the others?

Representing edges from multiple layers for large graphs induces highly cluttered visualizations. Grouping edges into bundles is a successful method to reduce edge cluttering in graphs $[5,10,14,13$, $23,8,16,11,3]$. Preliminary works on edge bundling also include techniques dealing with various kinds of graph, such as compound graphs [9] or directed graphs [21]. Unfortunately, previous works on multilayer graph visualization $[1,12,4,7,6,19,20,17]$ did not consider edge bundling approaches.

Applying standard edge bundling to multilayer graphs does not supply suitable results. Edges from different layers can be grouped together reducing the possibility to highlight patterns specific to a particular layer or, conversely, patterns shared among different layers. This fact motivates our research. In this paper, we propose a new edge-bundling technique that avoids to group edges of different layers. The result of our technique is a visualization in which a single map captures similarities and differences of edge distribution among layers.

More in detail, our proposed technique has four main steps: firstly it adapts the technique proposed in [14] to obtain a preliminary edge bundling (Section 2). Secondly, starting from the preliminary edge bundling, our framework smooths the edge visualization (Section 3). Successively, it divides the previously obtained bundle in layer specific bundles enforcing each bundle to contain only edges belonging to the same layer (Section 4). Finally, as the layer specific bundles can cross each others, we reduce the number of inter bundles crossing (Section 5).

\section{INITIAL Edge BundLING}

We start to define some basic elements we use in the rest of the paper. The terms node and edge refer to multilayer graph node and edge. A control point is a vertex employed to route the edges between two nodes while a segment is a line between two control points over which more than one edge can pass through. A path is a set of consecutive segments between a pair of nodes.

As first step, our approach considers the multilayer graph as a simple graph where no distinction between edge layers is made. We name it flattened graph. The flattened graph has as many nodes as the original multilayer graph and if two nodes are linked in any of the layers of the multilayer graph, then an edge will exist in the flattened graph. The flattened graph is employed to obtain a preliminary edge bundling. To perform such bundling we adapt the algorithm Winding Roads proposed in [14]. Starting from a graph with predefined node positions, this algorithm performs edge bundling discretizing the space around nodes considering a mix of Voronoi diagram and Quad-tree. Instead of employ the algorithm as it was proposed, we only consider Voronoi diagram to perform the space discretization as we observed that directly apply the Winding Roads algorithm, as it is, results in an over-discretization of the space that negatively impacts the final result. The initial edge bundling step supplies a grid, derived by the Voronoi diagram, where the vertex of the grid are the control points and the lines between control 


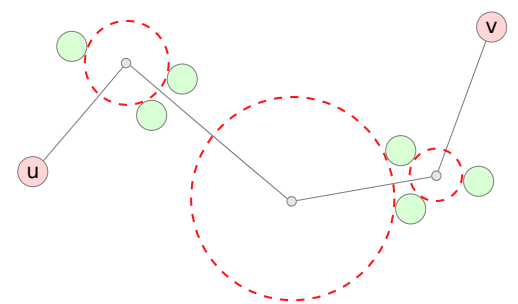

(a)

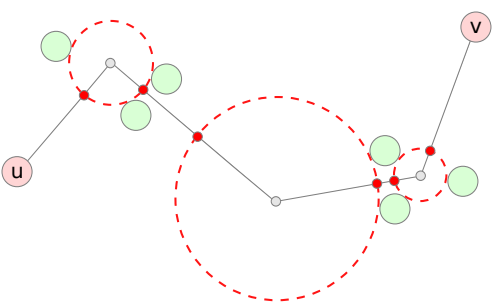

(b)

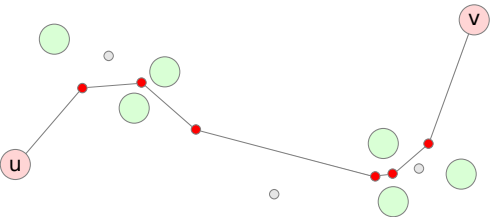

(c)

Figure 1: Edge Smoothing procedure: a) computation of the free space around control points b) determination of the new control points c) the new control points (in red) are added to the set of already existing control points, and new segments are added to route the edges.

points are the segments over which more than one edge can pass through. The segments are successively used to route the edges of the multilayer graph into bundles.

\section{Edge SMOOTHING}

As we explained before, to route an edge we employ a set of consecutive segments that link two nodes of the multilayer graph. The same segment can be traversed by more than one edge type and smoothing such set of segments can be helpful to improve the final visualization. In order to better draw the edges between two nodes we propose an Edge Smoothing procedure that firstly determines the amount of free space around a control point and then creates new control points (and segments) to smooth the trajectory of the edges.

\subsection{Determine free space around control points}

The first step of the Edge Smoothing procedure is dedicated to understand how much free space is available around each control point in order to avoid overlap between edges and nodes in the multilayer graph visualization. This free space is quantified by a radius around the control points and such radius is represented by the distance between the control point and its closest nodes (see Figure 1a and the red dotted circles).

\subsection{Create new control points}

Given a control point $x$, once the radius $d$ corresponding to the free space around it is determined, we can create new control points considering points at distance $d$ on the adjacent segments to $x$ (see red points in Figure 1b). Once the new control points are obtained, the curve can be smoothed adding new segments between new control points as depicted in Figure 1c. We distinguished the new control points w.r.t. the previous ones by a different color. More in detail, we used the red color for the new control points and the gray color for the control points that already exist in the initial Voronoi grid. As a result, we obtain smoother bundles than the previous ones.

If the smoothing result needs to be ameliorated, we can repeat the previous process recomputing the radius related to the available space around each control point and, then, create new control points. This procedure ensures that, if there was no overlap before between nodes and edges then the Edge Smoothing step will not introduce any of them. This is due to the locations where the new control points are placed and to the fact that the radius related to each control point is computed considering its closest node. Repeating the smoothing procedure can lead to better visualization but it will increase the computational time.

\section{Per layer Bundle Division}

At this point, the different types of edges can be routed through the set of control points and segments. Unfortunately, the obtained bundles are not specific for a layer, this means that edges coming from different layers can overlap each others. To tackle this issue, we propose to divide each bundle of the flatten graph to obtain different bundles, one for each edge type traversing the corresponding bundle of the flattened graph. To do this, firstly we determine the free space around each control points (in the same way as done in Section 3.1). Then, we break the bundle splitting it into as many bundles as the different types of edges it contains. The proposed approach does not really avoid overlap between edges and nodes as shown in Figure $2 \mathrm{~b}$ where violet segment overlaps green node. For this reason we propose, later, a way to overcome this issue in Section 4.2.

\subsection{Bundle Division}

In this section we use the similar notation we employ in Section 3.2 to explain how we divide the bundle. Considering Figure 2a, for each new control point (red points) we compute again the distance between it and the closest node to determine the circle corresponding to its available free space (see dotted red circle in Figure 2a).

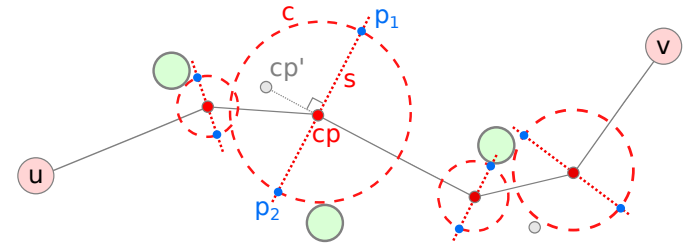

(a)

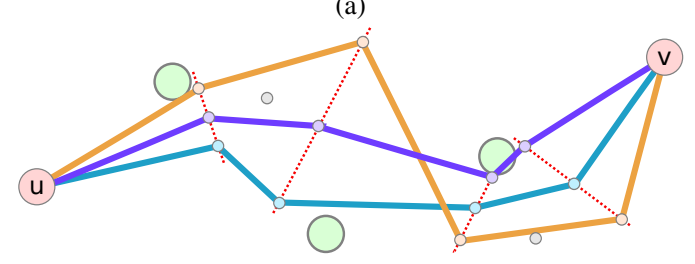

(b)

Figure 2: Per Layer Bundle Division: (a) compute the perpendicular segment to the control point considering control points that already exist (b) per layer division of control points.

Then, for each control point we determine a segment that pass through it and intersects the circle determining the free space. For instance, in Figure $2 \mathrm{a}$ we can observe that the control point $c p$ is surrounded by the circle $c$. Considering $c p$ and $c$, we draw the segment $s$ that intersects $c$ at the points $p_{1}$ and $p_{2} . s$ is drawn perpendicular to the segment linking $c p$ and the gray control points from which it is generated. In the example in Figure $2 \mathrm{a}$, the segment $s$ is perpendicular to the segment $\left[c p, c p^{\prime}\right]$. Successively, we duplicate the control point $c p$ into several control points, one for each edge type of the bundle. Finally, we locate the new control points uniformly on $s$. Once this operation is done, for each original control point of the bundle, we use the new control points to draw the different edge types without a particular order (see Figure $2 b$ ). 


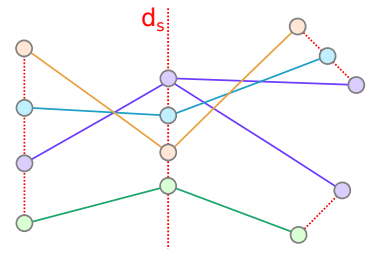

(a)

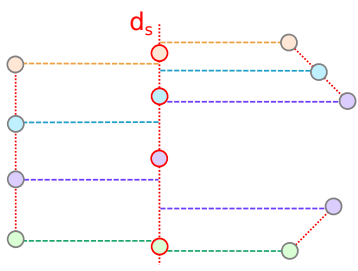

(b)

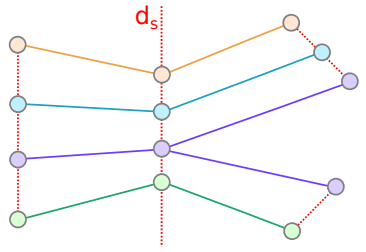

(c)

Figure 3: Bundle Crossing Reduction heuristic: a) initial scenario with bundle crossing b) computation of the barycenters of the projections of the control points neighbors on $d_{s}$ (barycenters are depicted with a red border) c) new order of the control points on $d_{s}$.

\subsection{Avoiding Edge-Node Overlap}

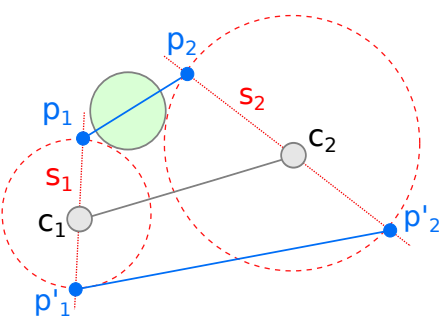

(a)

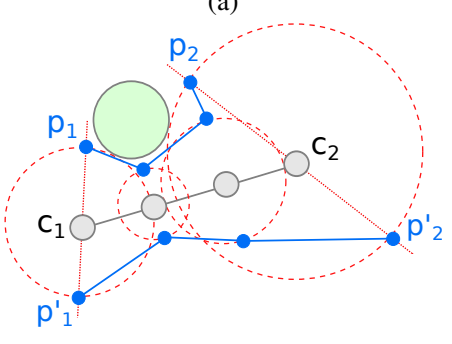

(b)

Figure 4: Edge-Node overlap heuristic: (a) the issue we can have drawing the per-layer bundles b) the over-discretization heuristic (with $K=3$ ) we employ to deal with the edge-node overlap problem.

In a more general scenario, if we consider only the space around the control points to draw the different edge types we can fall in a situation similar to the one reported in Figure $2 b$ (violet segment overlaps green node). As we can observe, one or more of the new segments can overlap the nodes inducing ambiguity in the visualization. In order to address this issue, we adopt the following strategy, named Edge-Node overlap heuristic: let $s_{1}$ (resp. $s_{2}$ ) be the segment incident on control point $c_{1}$ (resp. $c_{2}$ ) as explained in Section 4.1 and, $p_{1}$ and $p_{1}^{\prime}$ (resp. $p_{2}$ and $p_{2}^{\prime}$ ) be the endpoints of $s_{1}$ (resp. $s_{2}$ ). Then if a node $n$ falls into the polygon defined by the set of points $\left(p_{1}, p_{2}, p_{2}^{\prime}, p_{1}^{\prime}\right)$ (see Figure $4 \mathrm{a}$ ) we over-discretize the segment $\left[c_{1}, c_{2}\right]$ in $K$ segments adding $K-1$ control points uniformly distributed along its length. Such a trick helps to deal with the overlap issue since new control points are added to refine the bundle division step introduced in Section 4.1 (see Figure 4b). In our approach we fix $K=10$ as we empirically observe that this number supplies a good trade off between computational complexity and visual result.

\section{BUNDLE CROSSING REDUCTION}

As shown in Figure 3a, the previous steps of our framework can induce edge crossing between edges of different types, affecting the multilayer graph visualization. This phenomenon happens because consecutive control points, traversed by the same type of edge, could not have the same order on their segment. For instance, we can observe in Figure 3 a that control points employed to draw the orange bundle do not have the same relative position along the different segments. To deal with this issue, we adapt the barycenter heuristic, usually employed to draw DAGs [22]. More in detail, in our case we want to find the order of the control points on the line $d_{s}$ that minimizes the number of crossing edges. This problem is closely related to the metro-line crossing minimization task [18]. Alternative heuristics have been proposed in [18] and they could be also adapted to our problem.

Given the line $d_{s}$ and a set of control points lying on it, for a control point of a particular edge type we compute the barycenter of the projections of its neighbors on $d_{s}$. We repeat the same procedure for all the control points lying on $d_{s}$. Considering our example in Figure 3a, Figure 3b shows the barycenter computed for each control point on $d_{s}$ considering its neighbors. The barycenters are highlighted by points on $d_{s}$ with red border. Then, the control points of $s$ are reordered according to the computed barycenter obtaining a new order of the original control points as depicted in Figure 3c.

\section{Case Studies}

In this section we illustrate a case study that shows the practical benefits of our method to visualize multilayer graph data. The case study investigates social interaction among people who communicate through different media. We employ the Reality Mining dataset $^{1}$. This multilayer graph contains human interaction data collected by the MIT Media Lab. The experiment was carried out on a total of 94 people and this also represents the number of nodes in the corresponding multilayer graph. The different layers offered by the dataset pertain to the means of interaction between a pair of people. Namely, CALL layer refers to subjects calling each other, FRIEND layer contains friendship claims, SMS layer builds on text message exchanges (SMS) and DEVICE layer contains Bluetooth device scans. For our purpose we consider the first three layers (CALL, FRIEND and SMS) discarding the DEVICE layer as it is a quasi-clique and it does not provide useful information in the context of edge bundling visualization. The three considered layers have, respectively, 177, 82 and 113 edges. Experiments are carried out on an Desktop Computer with Intel Core i7-3770 CPU @ 3.40 $\mathrm{GHz} \times 8$, with $8 \mathrm{~Gb}$ of RAM.

Figure 5 shows the result of our multilayer graph edge bundling on the portion of Reality Mining dataset we consider. The violet edges correspond to the CALL layer, the green edges correspond to the FRIEND layer and SMS layer is depicted in orange. Curved edges that are not parts of bundles are artifacts of the initial bundling algorithm (see Section 2). Computation time is of 2.40 seconds with 2 iterations of the edge smoothing process.

Analyzing the visual result, we can note that the graph have three cluster structures: $C_{1}, C_{2}$ and $C_{3}$. We can observe that $C_{1}$ and $C_{2}$ contain edges of different types while $C_{3}$ contains only people that

\footnotetext{
${ }^{1}$ http://realitycommons.media.mit.edu/ realitymining.html [Online; accessed 12-March-2015]
} 


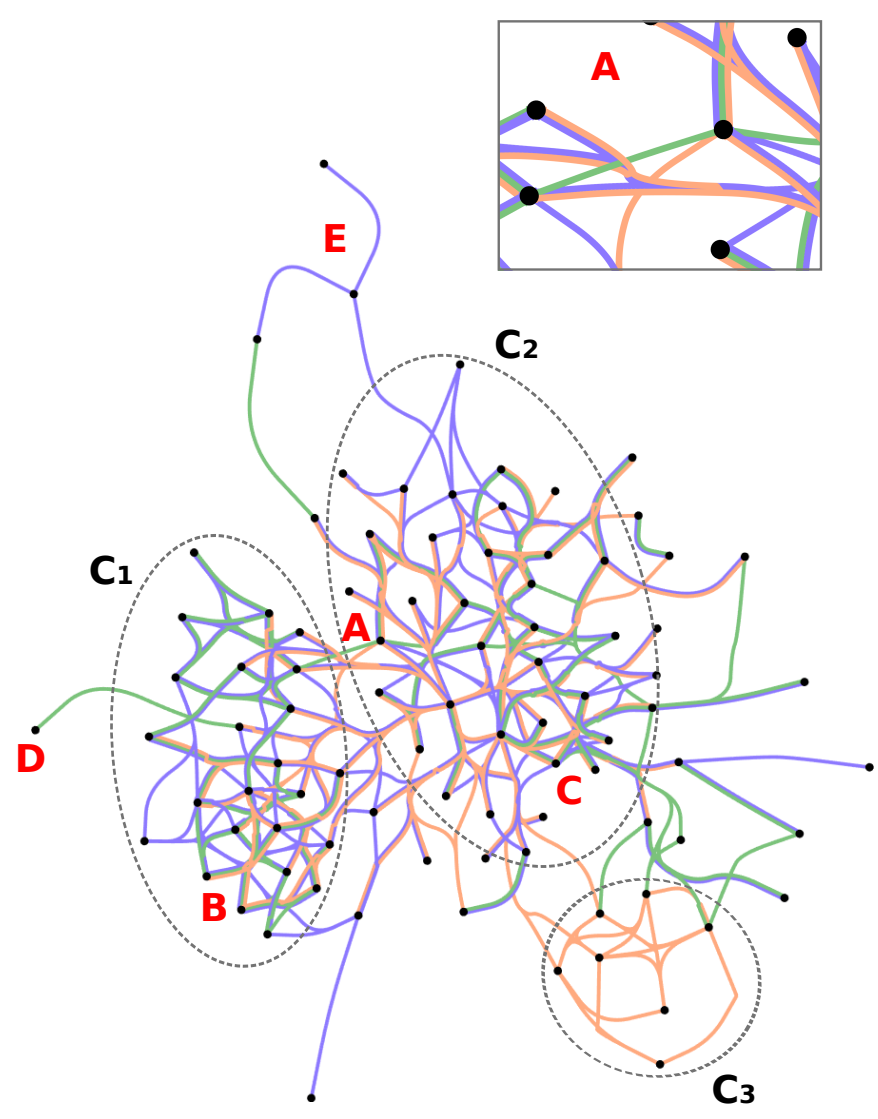

and interact each other only through CALL (violet layer) while a different behavior is depicted at the core of $C_{1}$ where people that are friends (green layer) communicate with both mobile CALL (violet layer) and SMS (orange layer) as shown in Figure 5 letter (B). In the same way (Figure 5 letter (C)), the core part of $C_{2}$ contains people in a friend relationship (green layer) that communicate similarly as done by the core of community $C_{1}$. A different behavior is shown by community $C_{3}$ where people belonging to this cluster only communicate each other with SMS (orange layer).

The visualization allows also to identify some kind of anomalies in the graph structure and the information about the different layers can help to analyze such anomalies. For instance, considering the left part of the graph (see Figure 5 letter (D)), we can observe that an isolate node is connected to community $C_{1}$ and this (green) link underlines that this outlier node is a friend of a people belonging to $C_{1}$. Another anomalous behavior is shown in the top of the graph (Figure 5, letter (E)) where, two nodes, that do not belong to any cluster, communicate with other people only through mobile CALL (violet layer). We can found a similar pattern on the extreme right and at the bottom of the multilayer graph. We can consider these nodes as anomalies showing a similar communication pattern. All of them, to some extent, represent community outliers that interact with other people only through mobile CALL (violet layer).

To the sake of completeness, we also evaluate our multilayer graph edge bundling on a bigger multilayer graph composed by 301 nodes and 3326 edges (considering all the layers). This multilayer graph is a subsample of the BIOGRID dataset [2], a protein-protein interactions network where nodes represent proteins and edges represent interactions between proteins. This multilayer graph contains 8 layers. Figure 6 shows the visualization of the BIOGRID multilayer graph before (Figure 6a) and after (Figure 6b) our approach is applied. Computation time is 1037.12 seconds with 1 iteration of the edge smoothing process. Also in this case, we can observe that our proposal firstly helps to better emphasize the global multilayer graph structure and secondly it still allows to minimize the edge cluttering issue especially among edges of different type.

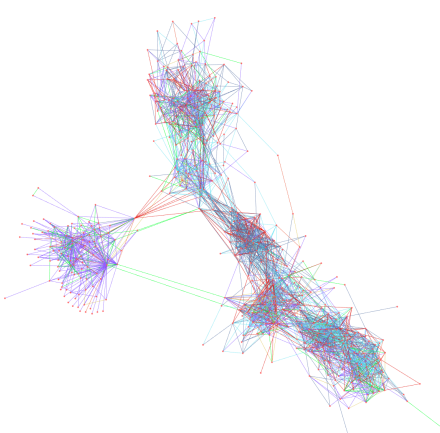

(a)

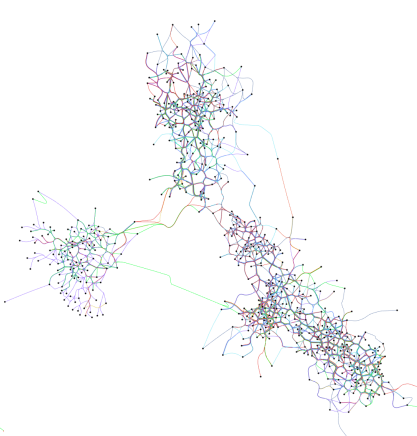

(b) layer) without necessarily being friends. There is only a green link between this two communities highlighting a friend relationship that constitutes an isolate case or an abnormal interaction behavior between $C_{1}$ and $C_{2}$ (see Figure 5, letter (A) ). Considering the way in which people belonging to $C_{2}$ are linked with people in the community $C_{3}$, we can note that some of them are involved in a friend relationship (green layer) while others employ SMS (orange layer) to interact. What is highlighted by the visualization is that no mobile CALL (violet layer) are performed between community $C_{2}$ and $C_{3}$. The visualization also supplies the information that no interactions between the communities $C_{2}$ and $C_{3}$ exist.

The multilayer graph edge bundling technique also helps to highlight behaviors that are specific to a portion of the graph. For instance, considering cluster $C_{1}$, we can note that, at the top of this community, there is a group of people that are friends (green layer)

Figure 6: Visualization of the subsample of BIOGRID multilayer graph: a) the visualization before applying our approach b) the result obtained with the multilayer graph edge bundling strategy.

\section{CONCLUSION AND FUtURE WORK}

In this paper, we have presented a novel and intuitive technique to route different types of edges into bundles to visualize multilayer graphs. Our approach reduces edge clutter at both global and per layer level. As future work, we plan to consider the number of edges passing through a bundle to determine its width and manage weighted multilayer graph. 


\section{REFERENCES}

[1] T. Bläsius, S. G. Kobourov, and I. Rutter. Simultaneous embedding of planar graphs. In Handbook of Graph Drawing and Visualization, page 349383. CRC Press, 2013.

[2] F. Bonchi, A. Gionis, F. Gullo, and A. Ukkonen. Distance oracles in edge-labeled graphs. In Proceedings of the International Conference on Extending Database Technology (EDBT), pages 547-558, 2014.

[3] Q. W. Bouts and B. Speckmann. Clustered edge routing. In Proceedings of the IEEE Pacific Visualization Symposium (PacificVis), pages 55-62, 2015.

[4] T. Crnovrsanin, C. Muelder, R. Faris, D. Felmlee, and K.-L. Ma. Visualization techniques for categorical analysis of social networks with multiple edge sets. Social Networks, 37:56-64, 2014.

[5] W. Cui, H. Zhou, H. Qu, P. C. Wong, and X. Li. Geometry-based edge clustering for graph visualization. IEEE Transactions on Visualization Computer Graphics, 14(6):1277-1284, 2008.

[6] M. D. Domenico, M. A. Porter, and A. Arenas. MuxViz: a tool for multilayer analysis and visualization of networks. Journal of Complex Networks, 3:159-176, 2014.

[7] S. Elzen and J. van Wijk. Multivariate network exploration and presentation: From detail to overview via selections and aggregations. IEEE Transactions on Visualization and Computer Graphics, 20(12):23102319, 2014.

[8] O. Ersoy, C. Hurter, F. V. Paulovich, G. Cantareiro, and A. Telea Skeleton-based edge bundling for graph visualization. IEEE Transactions on Visualization Computer Graphic, 17(12):2364-2373, 2011.

[9] D. Holten. Hierarchical edge bundles: Visualization of adjacency relations in hierarchical data. IEEE Transactions on Visualization Computer Graphics, 12(5):741-748, 2006.

[10] D. Holten and J. J. van Wijk. Force-directed edge bundling for graph visualization. Computer Graphics Forum, 28(3):983-990, 2009.

[11] C. Hurter, O. Ersoy, and A. Telea. Graph bundling by kernel density estimation. Computer Graphics Forum, 31(3):865-874, 2012.

[12] A. Kerren, H. Purchase, and M. O. Ward. Information visualizationtowards multivariate network visualization (dagstuhl seminar 13201). Dagstuhl Reports, 3(5), 2013.

[13] A. Lambert, R. Bourqui, and D. Auber. 3D edge bundling for geographical data visualization. In Proceedings of the 14th International Conference on Information Visualisation (IV), pages 329-335, 2010.

[14] A. Lambert, R. Bourqui, and D. Auber. Winding roads: Routing edges into bundles. Computer Graphics Forum, 29(3):853-862, 2010.

[15] J. Leskovec, A. Singh, and J. Kleinberg. Patterns of influence in a recommendation network. In Proceedings of the Pacific-Asia Conference on Knowledge Discovery and Data Mining (PAKDD), pages 380-389, 2006.

[16] S.-J. Luo, C.-L. Liu, B.-Y. Chen, and K.-L. Ma. Ambiguity-free edgebundling for interactive graph visualization. IEEE Transactions on Visualization Computer Graphic, 18(5):810-821, 2012.

[17] A. Meidiana and S.-H. Hong. MultiStory: Visual analytics of dynamic multi-relational networks. In Proceedings of the IEEE Pacific Visualization Symposium (Pacific Vis), pages 75-79, 2015.

[18] M. Nöllenburg. An improved algorithm for the metro-line crossing minimization problem. In Proceedings of the Conference on Graph Drawing (GD'09), volume 5849 of Lecture Notes in Computer Science, pages 381-392. Springer, 2010.

[19] D. Redondo, A. Sallaberry, D. Ienco, F. Zaidi, and P. Poncelet. Layercentered approach for multigraphs visualization. In Proceedings of the 19th International Conference on Information Visualisation (IV), pages 50-55, 2015.

[20] B. Renoust, G. Melançon, and T. Munzner. Detangler: Visual analytics for multiplex networks. Computer Graphics Forum, 34(3):321330, 2015.

[21] D. Selassie, B. Heller, and J. Heer. Divided edge bundling for directional network data. IEEE Transactions on Visualization Computer Graphics, 17(12):2354-2363, 2011.

[22] K. Sugiyama, S. Tagawa, and M. Toda. Methods for visual understanding of hierarchical system structures. IEEE Transactions on Systems, Man, and Cybernetics, 11(2):109-125, 1981.

[23] A. Telea and O. Ersoy. Image-based edge bundles: Simplified visu- alization of large graphs. Computer Graphics Forum, 29(3):843-852, 2010.

[24] A. Zhang. Protein Interaction Networks: Computational Analysis. Cambridge University Press, 2009. 